EPiC Series in Health Sciences
Volume 3, 2019, Pages 436-439
$\begin{aligned} & \text { CAOS 2019. The 19th Annual Meeting of the International } \\ & \text { Society for Computer Assisted Orthopaedic Surgery }\end{aligned}$

\title{
Robotic-arm assisted total knee arthroplasty improves precision and delivers early outcomes superior to manual approach
}

\author{
Jenny Zhang, BA, ${ }^{1}$ Nipun Sodhi, MD,${ }^{1}$ Kristina Dushaj, MA, ${ }^{1}$ \\ Michael Mont, $\mathrm{MD}^{1}$ and Matthew Hepinstall, $\mathrm{MD}^{1}$ \\ ${ }^{1}$ Department of Orthopedics, Lenox Hill Hospital, New York, NY \\ JZhang41@northwell.edu
}

\begin{abstract}
Haptic robotic-arm assisted technology improves accuracy in unicompartmental knee replacement through utilizing a preoperative 3-D plan, optical navigation for real-time intraoperative feedback on soft tissue laxity, and robotic arm for precise bone preparation. This technology became clinically available for total knee arthroplasty (TKA) in 2016. We present outcomes from the early adoption of this technique.

A retrospective chart review compared data from the first 120 robotic-arm assisted TKAs performed December 2016 through July 2018 to the last 120 manually instrumented TKAs performed May 2015 to December 2016, prior to robotic technology adoption.

Robotic surgery was associated with significantly increased anesthesia (212 vs 187 mins, $p<0.01)$ and operative ( 135 vs 112 minutes, $p<0.01)$ time. The robotic group had a lower hospital length-of-stay ( 2.7 vs. 3.4 days, $\mathrm{p}<0.001)$. Discharge to home was not statistically different between robotic and manual groups $(89 \%$ vs. $83 \%, p=0.2)$. Robotic technology was associated with decreased variability in implant positioning, with smaller variances in the lateral distal femoral angle (LDFA; 3.5 vs 6.6 degrees, $p<0.01)$ and posterior tibial slope ( 1.8 vs. 5.3 degrees, $\mathrm{p}<0.01)$. Mean limb alignment, as measured by tibiofemoral angle, was slightly less valgus in the robotic group (3.9 vs 4.4 degrees, $p$ $=0.09$ ). Postoperative range of motion was significantly increased for robotic-arm assisted TKA patients, with less flexion contracture at 2 -weeks (1.8 vs. 3.3 degrees, $\mathrm{p}<$ 0.01 ), 7 -weeks ( 1.0 vs. 1.8 degrees, $p<0.01$ ), and 3 -months ( 0.6 vs 2.1 degrees, $p=0.02$ ) post-surgery. Postoperative Knee Society scores were similar between groups.

Preliminary findings demonstrate robotic-arm assisted TKA is safe and efficacious with outcomes comparable, if not superior, to that of manually instrumented TKA.
\end{abstract}




\section{Introduction}

Despite innovations in implant design and instrumentation, conventional, manually instrumented total knee arthroplasty (TKA) is associated with technical errors of malalignment, implant malpositioning, and ligament imbalance; this can lead to knee instability, early failure, reoperation, and decreased patient satisfaction [1-3]. Haptic roboticarm assisted technology improves accuracy in unicompartmental knee replacement (UKA) through utilizing a preoperative 3-D plan, optical navigation for real-time intraoperative feedback on soft tissue laxity, and robotic arm for precise bone preparation [4]. This technology became clinically available for TKA in 2016. Therefore, the purpose of this study is to present early outcomes associated with the robotic-arm assisted TKA procedure and compare them to the manually instrumented approach. Specifically, we evaluated: (1) operative time and relevant perioperative outcomes, (2) accuracy of component placement, and (3) recovery of knee function after primary TKA.

\section{Methods}

This study retrospectively reviewed data from the first 120 robotic-arm assisted, unilateral TKAs performed at our institution from December 2016 to July 2018 (the "robotic" group) and the last 120 manually instrumented TKAs done from May 2015 to December 2016, prior to adoption of robotic technology, (the "manual" group). Manual and robotic surgeries were performed with the same standardized perioperative protocol, including the use of pneumatic tourniquets from exposure through cementation and typically until wound closure, but not for more than 120 minutes. The prosthetic components differed between groups. The manual group included cruciate retaining, posterior stabilized and constrained condylar variants of three brands: Persona (Zimmer, Warsaw, Indiana), Legion (Smith \& Nephew) and Journey 2 (Smith \& Nephew) whereas all patients in the robotic group received cruciate retaining, posterior stabilized and constrained condylar variants of the implant supported by the robotic system, Triathlon (Stryker, Kalamazoo, MI). Standardized discharge criteria were used in both groups.

Patient demographic data (age, gender, BMI) and preoperative diagnoses were recorded and compared between cohorts. The original Knee Society Knee Scores (KSS) were computed to assess joint functionality at 2-weeks, 7-weeks, and 3-months post-surgery. Anesthesia and operative time (from incision to end of closure), length of stay, discharge disposition, blood transfusions, manipulations under anesthesia, and postoperative complications were noted. To assess implant placement and limb alignment, we measured the anatomic tibiofemoral angle (TFA), lateral distal femoral angle (LDFA), medial proximal tibial angle (MPTA) on AP radiographs and posterior slope of the proximal tibia on lateral radiographs [5]. 


\section{Results}

Demographic data between groups did not differ with the exception of gender; the manual group had $76 \%$ women versus $58 \%$ women in the robotic group $(\mathrm{p}=0.004)$. Robotic surgery was associated with significantly increased anesthesia (212 vs 187 mins, $p<0.01)$ and operative $(135$ vs 112 mins, $\mathrm{p}<0.01)$ time. However, there was no difference in intraoperative blood loss as measured by hemoglobin drop and there were no packed red blood cell transfusions in either group. The average operative time for the last ten robotic cases was 26 minutes shorter than the first ten cases, indicating the presence of a learning curve for efficiency. The robotic group had a lower hospital length-of-stay ( 2.7 vs. 3.4 days, $\mathrm{p}<0.001)$. Discharge to home was higher in the robotic group, but the difference was not statistically significant ( 89 vs. $83 \%, p=0.20$ ).

Table 1: Intraoperative and early post-operative outcomes

\begin{tabular}{|l|l|l|l|}
\hline & Robotic & Manual & p-value \\
\hline Anesthesia Time (mins), mean (SD) & $212(32)$ & $187(28)$ & $<0.001^{*}$ \\
\hline Operative Time (mins), mean (SD) & $135(26)$ & $112(21)$ & $<0.001^{*}$ \\
\hline Hemoglobin Drop, mean (SD) & $1.7(1.0)$ & $1.6(0.8)$ & 0.732 \\
\hline Length of Stay (days), mean (SD) & $2.7(1.4)$ & $3.4(1.7)$ & $<0.001^{*}$ \\
\hline Discharge to Home, n (\%) & $107(89)$ & $99(83)$ & 0.195 \\
\hline
\end{tabular}

Radiographic measurements showed robotic technology decreased variability in implant positioning with fewer outliers. The robotic group had smaller variances in LDFA (3.5 vs $6.6, \mathrm{p}<0.01)$ and posterior tibial slope (1.8 vs 5.3, $\mathrm{p}<0.01)$. Mean limb alignment, as measured by tibiofemoral angle, was slightly less valgus in the robotic group (3.9 vs 4.4 degrees, $p=0.09$ ). Postoperative range-of-motion was significantly increased for roboticarm assisted TKA patients, with less flexion contracture at 2-week (1.8 vs 3.3 degrees, $\mathrm{p}<$ $0.01)$, 7-week (1.0 vs 1.8 degrees, $\mathrm{p}<0.01)$, and 3 -month $(0.6$ vs 2.1 degrees, $\mathrm{p}=0.024)$ follow-up. Furthermore, patients who underwent robotic TKA had significantly fewer manipulations under anesthesia ( $4 \%$ vs $17 \%, p=0.013)$. Postoperative Knee Society knee scores were similar between groups at 2 -weeks ( 63 vs. 62 points, $p=0.85)$, 7-weeks ( 69 vs. 66 points, $\mathrm{p}=0.48$ ), and 3 -months ( $72 \mathrm{vs.} 71$ points, $\mathrm{p}=0.80$ ) post-surgery.

\section{Discussion \& Conclusion}

This study is one of the first to investigate and compare intraoperative and short-term postoperative outcomes in TKA performed via a haptic robotic-arm assisted versus manual 
approach. Preliminary outcomes demonstrate robotic-arm assisted TKA is safe and efficacious with outcomes comparable, if not superior, to that of manually instrumented TKA. The technology offers more precision compared to the conventional approach and is associated with more ideal component positioning and alignment, lower variability, and smaller frequency of outliers. These findings validate manufacturer claims and are consistent with the literature evaluating the use of robotic-arm assisted technology in UKA [6-7]. In addition, these improvements can be translated to better, more consistent early clinical outcomes. Robotic technology was associated with faster recovery, as demonstrated by a statistically significant lower length of stay, and increased range of motion post-operatively.

Limitations of this study include its retrospective nature as well as its relatively short follow-up period. Patients were not randomized, and clinical assessment was not blinded. Gender differed between groups and, although other demographic variables were comparable between groups, this may introduce bias. Given the sequential study design, we acknowledge certain improvements in discharge disposition or length of stay could be the result of cultural shifts related to discharge. Implants also differed between groups, as the surgical robot currently only supports a specific implant. The observed improved early outcomes in the robotic group includes the learning curve or adoption phase for both the implant and the robotic technology, which would have been expected to bias the results in favor of the manual technique. It is reassuring therefore that all measured patient-centered outcomes were equivalent between groups or favored the robotic group, suggesting that robotic surgery does not compromise quality. Further work is needed to fully evaluate this robotic technology, including patient satisfaction, implant survivorship, and long-term outcomes.

\section{References}

1. Ferrara F, Cipriani A, Magarelli A et al. Implant positioning in TKA: Comparison between conventional and patient-specific instrumentation. Orthopedics. 2015 April;38(4):e271-e280.

2. Le DH, Goodman SB, Maloney WJ et al. Current modes of failure in TKA: infection, instability, and stiffness. Clin Orthop Relat Res. 2014 July;472(7):2197-200.

3. Meloni MC, Hoedemaeker RW, Violante B, et al. Soft tissue balancing in total knee arthroplasty. Joints. 2014 Jan-Mar;2(1):37-40.

4. Kleeblad LJ, Zuiderbaan HA, Hooper GJ et al. Unicompartmental knee arthroplasty: state of the art. JISAKOS 2017;2:97-107.

5. Paley D. Normal lower limb alignment and joint orientation. In: Principles of Deformity Correction. Springer-Verlag, Berlin, Heidelberg; 2002:1-17.

6. Blyth MJG, Anthony I, Rowe P et al. Robotic arm-assisted versus conventional unicompartmental knee arthroplasty: Exploratory secondary analysis of a randomised controlled trial. Bone Joint Res. 2017 Nov;6(11):631-9. 